\title{
Estimulación vestibular en el desarrollo infantil
}

\author{
Carla Solís-Gutiérrez *, Sandra Mariana Chávez Monjarás **, Víctor Manuel López Morales ***, \\ Cristina Carrillo-Prado ***, Jorge Alberto García-Martínez *****
}

\begin{abstract}
- Resumen
- El desarrollo infantil comprende la adquisición gradual de ha-

- bilidades en las áreas sensoriomotora, lenguaje y social, desde

- el nacimiento hasta los 12 años; éstas son mediadas por la

- maduración de estructuras del sistema nervioso que permiten

- al niño relacionarse con su entorno. Lo anterior se lleva a cabo

- de manera eficaz cuando existe un óptimo procesamiento

- sensoriomotor, en donde el sistema nervioso central organiza

- e interpreta la información del exterior a través de los sentidos,

- y posteriormente ejecutar una respuesta adecuada. En esta lí-

- nea, la estimulación del sistema vestibular favorece al área de

- desarrollo motriz; sin embargo, también se ha comprobado que,

- a partir de esto, se benefician las otras áreas a través de las dos

- funciones básicas vestibulares (equilibrio y orientación espacial),

- lo que le posibilita al niño aprender, regular y adaptar el movi-

- miento, al transformar la información sensorial en respuestas

- posturales que promueven el comienzo de la verticalización del

- eje axial hasta consolidar la bipedestación, es decir, permiten al

- niño cumplir gradualmente sus necesidades de interactuar con

- el medio a través del movimiento en busca de nuevos apren-

- dizajes que conllevan a un desarrollo óptimo para su indepen-

- dencia. Este artículo tuvo como objetivo, a través de la revisión

- bibliográfica, enfatizar la importancia de la estimulación del sis-

- tema vestibular para la regulación del movimiento, favoreciendo

- el desarrollo infantil en sus diferentes áreas. LUXMÉDICA AÑo 14,

- NÚMERO 40, ENERO-ABRIL 2019 PP 41-51.
\end{abstract}

- Palabras clave: estimulación vestibular, desarrollo infantil, desempeño psicomotor.

\section{Abstract}

Child development includes the gradual acquisition of skills in the sensorimotor, language and social areas, from birth to 12 years; these are mediated by the maturation of structures of the nervous system that allow the children to relate to their environment. The above is carried out efficiently when there is optimal sensorimotor processing, where the central nervous system organizes and interprets the information from the outside through the senses, and then execute an adequate response. Thus, the stimulation of the vestibular system favors the area of motor development, however, it has also been proven that from this the other areas are benefited through the two basic vestibular functions (balance and spatial orientation); this allows the child to learn, regulate and adapt the movement, transforming sensory information into postural responses that promote the beginning of verticalization of the axial axis to consolidate standing, therefore, allowing the children to gradually meet their needs to interact with the environment through the movement in search of new learnings that lead to an optimal development of their independence. The objective of this article, through a literature review, is to emphasize the importance of the stimulation of the vestibular system for the regulation of movement, favoring child development in its different areas. LUXMÉDICA AÑO 14, NÚMERO 40, ENERO-ABRIL 2019 PP 41-51.

Keywords: vestibular stimulation, child development, psychomotor performance

* Estudiante de Licenciatura en Fisioterapia, Facultad de Enfermería. Universidad Autónoma de Querétaro.

** Licenciada en Fisioterapia. Maestría en Competencias Educativas. Facultad de Enfermería. Universidad Autónoma de Querétaro.

** Médico Especialista en Pediatría. Maestría en Psicopedagogía. Facultad de Enfermería. Universidad Autónoma de Querétaro.

Lic. en Psicología. Técnico Superior Universitario en Terapia Física. Maestría en Gestión Directiva en Sistemas de Salud. Escuela Nacional de Estu-

dios Superiores Unidad León.
Licenciado en Fisioterapia. Especialista en Salud Pública. Unidad de Investigación en Pediatría. Facultad de Enfermería. Universidad Autónoma de Querétaro.

Fecha de recibido: 4 de septiembre 2018

Fecha de aceptación: 12 de noviembre 2018

Correspondencia: Jorge Alberto García Martínez. Universidad Autónoma de Querétaro. Cerro de las Campanas sin número. Colonia Las Campanas. Código postal 76010. Querétaro, Querétaro. México.Correo electrónico: jorgea.gmfisio@gmail.com Teléfono: 00-52 (427)1482818 


\section{Introducción}

La intervención neurológica en la etapa pediátrica se basa en conceptos y trabajos destinados al desarrollo neuropsicológico que fundamenta las bases del desarrollo y neuroplasticidad; para ello, es importante, describir la relación de éstos con el sistema vestibular.

Según los periodos propuestos por Juan Delval sobre sobre los avances que se han hecho para estudiar a los niños y su desarrollo, desde la Primera Guerra Mundial hasta los años cincuenta es el periodo cuando se inician los trabajos teóricos más importantes de la psicología del desarrollo. Entre los autores de esta etapa destacan Piaget, Vigotsky y Wallon. ${ }^{1}$

Piaget propone la teoría del desarrollo cognitivo en la cual describe que, de manera general, en respuesta a una necesidad, interés o pregunta, el niño busca en su entorno elementos para crear conocimientos nuevos, construyendo una representación de la realidad y actuando con base en ella. En base a estos estipula 4 etapas graduales por las que transcurre el niño y en éstas hace énfasis sobre el desarrollo interno de las estructuras de la inteligencia., ${ }^{1,2}$

A diferencia de Piaget que considera el medio social como algo estable, Vigotsky hace énfasis en los mecanismos externos. Establece la importancia de la cooperación y del intercambio social en el desarrollo del niño y los considera indisolubles. Introduce el concepto de "Zona de desarrollo potencial o próximo", y la define como el nivel al que puede elevarse un individuo con ayuda de adultos o compañeros más capaces. ${ }^{1}$ Por su parte, Wallon, coincide con la idea de que el niño es un ser social, y además de los factores sociales, también considera los fisiológicos, biológicos y el movimiento como determinantes para el desarrollo del niño, definiendo el movimiento como la única expresión y primer instrumento de lo psíquico. $^{3}$

Ajuriaguerra, neuropsiquiatra y psicoanalista español, continuó impulsando la obra de Wallon, consolidando los principios y bases de la psicomotricidad. Remarcó la importancia de relacionar el comportamiento y el desarrollo del niño y la maduración de su sistema nervioso para la creación de estrategias educativas terapéuticas y rehabilitadoras adecuadas a las necesidades específicas del niño. ${ }^{3}$

Luria, discípulo de Vigotsky, revolucionó con sus obras, los conocimientos de la neurología y psicología. En 1973, propuso un modelo para comprender la relación entre el cerebro y el comportamiento, llamado "Modelo Laboral" (Working Brain) en el cual describe que cualquier actividad consiste en un sistema complejo de procesamiento simultáneo y concurrente a todos los niveles del cerebro; por ejemplo, la motricidad es indisociable a otros procesos, como de la organización del tono de reposo y acción, del control postural y de la regulación vestibular, de la noción del cuerpo con el espacio, memoria y de las aferencias del medio; esta visión permitió abordar la lesión cerebral y su rehabilitación, concluyen- 
do que una acción es distribuida en varias estructuras del cerebro. Una lesión tiende a producir un efecto de desorganización en el sistema y, por ende, en el comportamiento del niño. A través de la rehabilitación de la función se pretende llegar a la reorganización estructural. ${ }^{3}$ Igualmente Kennard, una neuróloga estadounidense, tuvo aportaciones en el estudio del desarrollo neuropsicológico. Abordó principalmente los efectos de una lesión neurológica en monos alrededor de los años treinta. Sus estudios condujeron a una de las primeras pruebas de los efectos de la edad sobre la neuroplasticidad, creando el principio de Kennard, que sugiere que un cerebro inmaduro debería ser capaz de recuperarse de una lesión a comparación de un cerebro más desarrollado., ${ }^{4,5}$

Por otra parte, en los años cincuenta se contaba con escaso conocimiento sobre el abordaje en niños con lesión neurológica, los tratamientos eran dirigidos a tratar aquella parte del cuerpo "afectada", que reflejaba los síntomas. Glenn Doman, Temple Fay, Berta y Karel Bobath, se percataron de la importancia de los factores de entrada (sensoriales o receptivos) en el funcionamiento del cerebro adulto, fundamentando la existencia de una información previa sobre la cual el hombre basa sus movimientos, concluyendo que, a medida que el cerebro madura, éste empieza a apreciar la correlación entre potencia motora y respuesta sensorial; ${ }^{6}$ de tal manera que el movimiento es resultado de una interacción dinámica entre la percepción, cognición y sistemas de acción, y resaltaron la habilidad del sistema nervioso central de recibir, integrar y responder al entorno para lograr un objetivo motor. ${ }^{7}$

En los años sesenta, Ayres, terapeuta ocupacional estadounidense, observó que los niños que se inquietaban por realizar simples tareas diarias, presentaban dificultad para la escritura y un bajo desempeño escolar. Concluyó que tenían un desorden neuronal que hacía ineficiente la organización de la información sensorial recibida por su sistema nervioso. ${ }^{8}$

Katona, en 1974, establece la importancia la estimulación del sistema vestibular junto con otros sistemas, a través de maniobras que hacen posible la aparición de aferencias que garantizan acciones motoras, en las cuales los neonatos activan la verticalización mientras la cabeza y el cuerpo se mueven debido a la estimulación evocada por los movimientos de aceleración o contra gravedad..$^{9-11}$

Estas contribuciones han permitido el desarrollo y aplicación de varios métodos de habilitación, facilitación y rehabilitación neurológica en pacientes pediátricos que utilizan como base diferentes formas de estimulación sensorial para mejorar el desarrollo neuromotor de bebés con factores de riesgo para daño neurológico y niños con retraso en el desarrollo. Una de las formas de estimulación sensorial más utilizada es la vestibular. Actualmente, con el uso de resonancia magnética funcional, es posible explorar la activación del cerebro cuando las señales visuales y vestibulares 
son complementarias, es decir, cuando indican que el individuo está en movimiento o estático con respecto a lo que le rodea; o cuando existe una disfunción que produce una falsa sensación de movimiento. ${ }^{12,13}$

Diferentes estudios de investigación en niños han comprobado, a través de la comparación de diversas formas de estimulación, que la vestibular es significativamente más efectiva por la relación neuroanatómica cercana del sistema vestibular con otros reguladores de funciones sensoriomotora. ${ }^{14}$ Esto impacta en la maduración y reorganización de sistema nervioso central del niño, que cuenta con mayores posibilidades de modificar su conducta gracias a la neuroplasticidad. ${ }^{15,16}$

El objetivo de esta revisión fue enfatizar en la importancia de la estimulación del sistema vestibular para la regulación del movimiento, favoreciendo el desarrollo infantil en sus diferentes áreas. La revisión bibliográfica se realizó con base en autores reconocidos en los diferentes temas de neuroanatomía y fisiología del sistema vestibular, fisioterapia pediátrica, neurohabilitación, neurodesarrollo neonatal e infantil, neuropsicología infantil, neuroplasticidad, integración sensorial e intervención en niños con lesión cerebral. Igualmente se incluyeron artículos donde se estudió la influencia que tiene el sistema vestibular sobre el control postural, equilibrio o movimiento; también se consideraron trabajos de investigación sobre la eficacia, efectividad o eficiencia de la estimulación vestibular, así como otros métodos de habilitación, facilitación y rehabilitación neurológica en el desarrollo infantil, sin importar las características del niño, es decir, niños sanos, con factores de riesgo para daño neurológico o alteraciones en el funcionamiento del sistema vestibular.

\section{DESARROLLO DEL TEMA}

\section{Funciones del sistema vestibular}

El sistema vestibular cumple con dos funciones básicas para lograr el control postural: el equilibrio y la orientación espacial; igualmente realiza la función de regulación del tono muscular. Esto se deriva del procesamiento sensorial, que consiste en recibir y utilizar información del exterior y el interior, lo que permite aprender, regular y adaptar el movimiento al transformar la información sensorial en respuestas motoras o posturales apropiadas. ${ }^{17}$

Los receptores vestibulares son aquellos que reciben la información del exterior, siendo éstos los órganos otolíticos (utrículo y sáculo) que responden a las aceleraciones lineales de la cabeza (flexión y extensión), y los canales semicirculares que responden a las angulares (rotación). La operación de éstos es un proceso complejo, ya que los diferentes tipos de entradas sensoriales se entremezclan en todo el cerebro ${ }^{8}$ a través de un conjunto de neuronas que van de un lugar del sistema nervioso a otro, formando tractos nerviosos. Las señales producidas por los receptores se transmiten a través del octavo par craneal hacia los núcleos vestibulares en el tallo cerebral, a la porción vestibular del cerebelo y a la porción de la corteza motora responsable de la coordinación y respuestas motoras, la po- 
sición de la cabeza, postura y movimiento de los ojos. ${ }^{18,19}$

Más específicamente, la estimulación de este sistema, a través de la vía vestíbuloocular y vestíbulo espinal, origina dos reflejos que sobresalen: el vestíbulo ocular y posturales vestibulares, su funcionamiento en conjunto produce las respuestas adaptativas del movimiento y cambios posturales de nuestro cuerpo hacia lo que nos rodea. De la misma manera, la interacción del sistema vestibular con el visual, con el estímulo de la fijación visual, son la fuente básica para poder estabilizar la cabeza en el espacio mientras se realiza alguna actividad. ${ }^{17,20}$

En la actualidad, la estimulación temprana infantil se define como el conjunto de acciones e intervenciones que favorecen el desarrollo del ser humano en sus primeras etapas de crecimiento. ${ }^{21}$ Es un aspecto que ha cobrado mucha importancia por su sólida base científica y por la estrecha relación con un neurodesarrollo exitoso.
El neurodesarrollo, va en consonancia con el desarrollo infantil. Estos procesos dinámicos se expresan a través de la interacción entre el niño y el medio que lo rodea; como resultado, se obtiene la maduración del sistema nervioso con la consiguiente adquisición de habilidades de las diferentes áreas: motoras gruesa y fina, la del lenguaje, sensorial y socialización; ${ }^{22}$ estas habilidades se obtienen a través del aprendizaje, la práctica y la experiencia.

Distintos autores han utilizado diagramas o esquemas para ilustrar el desarrollo humano. Lázaro y Berruezo, en el 2009, proponen una estructura piramidal profundizada en el esquema de Williams y Shellenberger para contemplar el desarrollo humano que sustenta el objetivo de este artículo. Esta estructura (figura I) contiene las capacidades del ser humano dispuestas en estratos de manera tal que, si existen dificultades en una capa superior, necesariamente hay que reforzar la inferior, y así sucesivamente hasta lo más básico ${ }^{23,24}$.

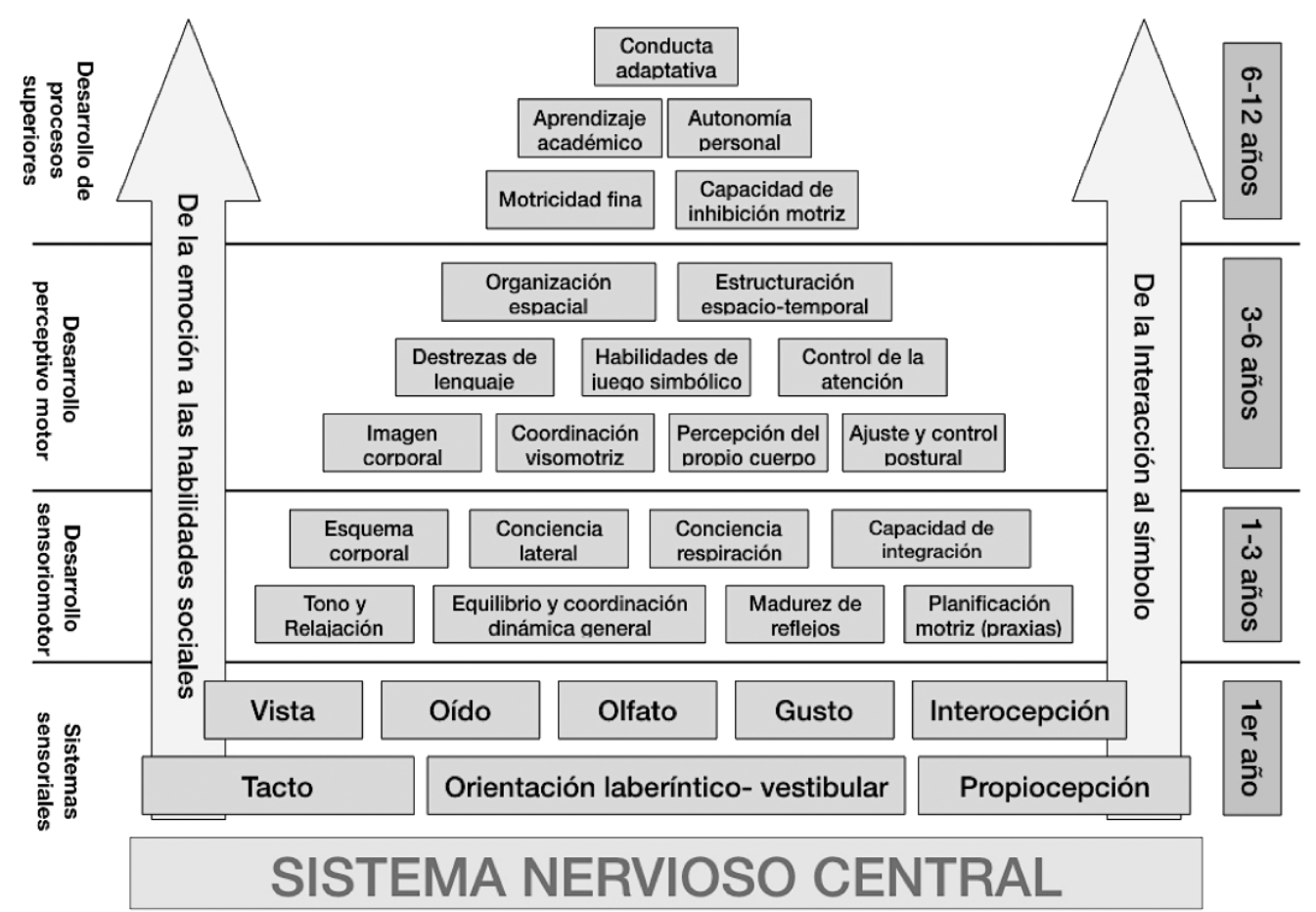

Figura 1. Tomada de Pirámide del desarrollo humano de Lázaro y Berruezo.24 
La base de la pirámide es el sistema nervioso central, específicamente el cerebro, que se encarga de integrar los sistemas sensoriales; de lo anterior se derivan $4 \mathrm{fa}$ ses, 10 niveles y dos ejes transversales.

La primera fase está constituida por los sistemas sensoriales. En el primer nivel se encuentran: el táctil, propioceptivo y vestibular; son la base fundamental del conocimiento propio y sobre el mundo. Éstos se han forjado a través de la filogénesis de la especie humana y la ontogénesis del individuo. Su estimulación surge de manera natural en la madre, padre o cuidador del niño, siendo un claro ejemplo cuando el adulto realiza un contacto físico con el niño al tocarlo o acariciarlo (estimulación táctil), sostenerlo en brazos (estimulación propioceptiva) y mecerlo (estimulación vestibular) para la contención del mismo. ${ }^{23}$

Como se observa en la segunda fase de la pirámide, el desarrollo sensoriomotor es clave para la adquisición de otras habilidades. Es por ello, que la intervención pediátrica tiene como base el conocimiento del desarrollo motor, dado que el movimiento es la respuesta a lo que se percibe en el entorno por medio de experiencias sensoriales, es decir, a través de los sentidos.

En un principio, el recién nacido posee escaso control postural y antigravitatorio, sus movimientos son globales y en masa ${ }^{20}$, lo que lleva al niño a desarrollarse en esta área, al ser su principal motivación el interés por alcanzar, tocar y realizar alguna actividad con objetos que le resultan llamativos. El desarrollo motor implica la búsqueda de una solución a una actividad determinada y emerge desde la interacción del individuo con la actividad del entorno. ${ }^{17}$

El sistema vestibular, al ser parte de la base de la pirámide, es fundamental para el desarrollo humano, es decir, si no se cuenta con un adecuado procesamiento del sistema nervioso central para recibir la información a este nivel, es imperativo trabajar en su integración para organizar la información y así dar una respuesta eficiente; de lo contrario, otros niveles se verán afectados, impidiendo un desarrollo óptimo del siguiente nivel. ${ }^{8}$

\section{Impacto funcional de las disfunciones vestibulares en el neurodesarrollo}

Las disfunciones en el sistema vestibular en los niños pueden originarse y estar asociadas a trastornos del desarrollo neurológico: trastornos de desarrollo intelectual, trastornos de comunicación, trastorno del espectro autista, trastorno por déficit de atención con hiperactividad, trastorno específico del aprendizaje, trastornos motores, descritos en el DSM- $5,{ }^{25}$ daños neurológicos ocasionados por factores biológicos, ambientales o sociales en el desarrollo pre, peri y postnatal; pérdida auditiva (ya que la cóclea está estrechamente ligada a este sistema); o por procesos de inflamación del oído medio manifestados comúnmente como vértigo. Éstos producen una sensación de movimiento del propio cuerpo o del ambiente circundante y pérdida del equilibrio. Es común encontrar que estas disfunciones retarden la sedestación y la postura bípeda en el primer año de vida e incluso puede acompañarse de dificultad en la fijación ocular al mover la cabeza. En niños mayores son comunes las caídas frecuentes, especialmente durante actividades motoras como brincar en un pie, caminar sobre superficies estrechas, entre otros. ${ }^{26,27}$ Al intervenir estas disfunciones, es posible corregir malas posturas, disminuyendo choques con objetos y caídas constantes. ${ }^{21}$ Igualmente, está comprobado que la pérdida completa de la función vestibular resulta en problemas en el aprendizaje por la poca referencia que tienen los niños de su propio cuerpo; se cree que en parte es porque la información propioceptiva sobre la gravedad no se ve reforzada por la sensación de gravedad otolítica, ${ }^{28,29}$ lo que provoca dificultad para comprender las relaciones espaciales (arriba-abajo, izquierda-derecha, adelante-atrás, adentroafuera, cerca-lejos, superior-inferior), esto implica desórdenes en la ejecución de sus praxis por la alteración o retraso en el de- 
sarrollo de la función vestibular, privando al niño de una retroalimentación sensoriomotora. Diferentes estudios comprueban que los niños que presentan esto se caracterizan por la dificultad en el proceso de lectura, escritura, conceptos numéricos, poco gusto por la participación en actividades deportivas, dificultades con la lateralidad, cruce de la línea media, coordinación bimanual, habilidades visoperceptuales y habilidades matemáticas. ${ }^{28,30}$ En Estados Unidos, más del $70 \%$ de los niños con dificultades para el aprendizaje en la escuela muestran una disfunción en la integración sensorial. ${ }^{31}$ Por lo anterior se observa que a través de la estimulación vestibular se procura una entrada sensorial a los niños, que les conduce a mayores niveles de alerta y activación emocional, contribuyendo al bienestar físico y psíquico, lo que permite al niño interactuar con su medio.

Intervención a través de la estimulación vestibular La prematurez es uno de los factores de riesgo con más incidencia en el país. Se estima que en México nacen anualmente 200 mil niños prematuros. ${ }^{32}$ Estos niños constituyen una población vulnerable sobre la cual se han hecho diversos estudios con la aplicación de la estimulación vestibular debido a que cursan con un riesgo elevado de presentar problemas de integración sensorial. Los problemas de procesamiento sensorial en prematuros se deben, en parte, a su estancia dentro de la Unidad de Cuidados Intensivos Neonatales, ya que el ambiente se considera limitado en estímulos, especialmente el vestibular, privando al lactante de recibir los estímulos que recibía in utero; siendo el sistema vestibular uno de los primeros sistemas sensoriales en desarrollarse, el prematuro puede ser más receptivo a su estimulación: existe literatura que comprueba la respuesta desde la semana 25 de gestación. ${ }^{11,33,34}$

En caso de que exista riesgo de daño, es posible intervenir y atenuar el efecto nocivo y llevar su desempeño psicomotriz lo más parecido a la normalidad gracias a la plasticidad cerebral. ${ }^{11,35}$ En sus primeros años, especialmente de los 0-2 años, las estructuras cerebrales están formándose y organizándose, ya que es una etapa especialmente sensible a las entradas del mundo exterior y esto puede ayudarnos a compensar los déficits mencionados o lesiones de estructuras cerebrales. ${ }^{36}$ El recién nacido tiene mayores posibilidades de modificar su conducta si son detectadas tempranamente las alteraciones con el fin de constituir un plan de intervención.

Respecto a la intervención en niños con discapacidades como trastornos del desarrollo neurológico, $X$ frágil, Angelman, entre otros, se ha observado que la estimulación vestíbulo- propioceptiva contribuye a la tranquilidad cuando es necesario lograr la calma, y la activación cuando se trata de aumentar el tono. ${ }^{23}$

Existen comportamientos autoestimulantes rítmicos que se puede observar en niños, especialmente diagnosticados con trastorno del espectro autista, que se caracterizan por la estimulación del movimiento (vestibular) impuesto externamente sobre el desarrollo conductual: es autoregulatorio. ${ }^{37}$ Entre éstas, las más comunes son el bodyrocking, el headbanging y headrolling, que como lo indican sus nombres en inglés, se refiere a cuando el niño mece su cuerpo, sacude su cabeza y realiza un movimiento tipo ondulatorio con la cabeza.

\section{Estimulación vestibular en el aula}

El reforzamiento de la estimulación vestibular en el aula lleva al niño a tener referencia sobre su propio cuerpo, lo cual favorece el aprendizaje de las relaciones espaciales. ${ }^{28,29}$ Esto conlleva a mejorar la ejecución de sus praxis, al generar una retroalimentación sensoriomotora, que a su vez beneficia el proceso de lectoescritura, conceptos numéricos, habilidades visoperceptuales y habilidades matemáticas. ${ }^{28,30}$ 
Otros beneficios comprobados sobre la estimulación vestibular La estimulación de la esfera motriz, a través del sistema vestibular junto con el propioceptivo, preside a los estados de activación motriz cortical para favorecer el desarrollo de la actividad psicológica general. Parte de la adquisición postural surgida durante la vida del niño, se da a partir del contacto e interacción con el adulto que lo facilita y lo propicia. Esto garantiza mejorar el estado emocional, al favorecer la aparición de las intenciones comunicativas e interactivas hacia acciones cada vez más complejas con su entorno..$^{10}$ Estudios comprueban la eficacia de la estimulación vestíbulo-propioceptiva para la contención del llanto en neonatos.

Otros beneficios que aporta este tipo de estimulación es la maduración de patrones de sueño de prematuros, ${ }^{38}$ ganancia ponderal de prematuros, ${ }^{34,39}$ alerta visual, ${ }^{34,39,40}$ contención de recién nacidos con y sin factores de riesgo, 10,34,39-41 reducción en los periodos de apnea y la regulación de la respiración, lo que a su vez proporciona una oxigenación sanguínea estable ayudando al lactante a ajustarse a las demandas de tareas como alimentación, primeras vocalizaciones, movimientos y cambios en la postura. ${ }^{33}$

En resumen, con los trabajos recolectados se obtuvo información teórica general sobre el tema de estimulación y desarrollo infantil, así como información específica de estudios e investigaciones sobre la estimulación vestibular a través de los años. Se sumó un total de 41 referencias que cumplieron con los criterios de inclusión para realizar este artículo. Cabe resaltar que, de los trabajos seleccionados, se observó una cantidad considerable de publicaciones a finales de la década de los setenta, siendo estos autores los pioneros de las principales aportaciones bibliográficas en incluir la estimulación sensorial y la actividad motora en un contexto terapéutico, por citar algunos, Doman en 1974, Katona en 1974, Bobath en 1975 y Ayres en 1979.

Los diferentes resultados descritos en los estudios revisados en este artículo permitieron realizar una tabla (Tabla 1) que sirve como referencia para conocer los beneficios comprobados que se pueden obtener a través de la estimulación vestibular. Ésta se divide en las edades propuestas en la pirámide del desarrollo (Figura 1) y al tipo de población a la que puede ser aplicada este tipo de estimulación según la edad. En cada una de las edades se mencionan los beneficios de la estimulación vestibular con base en la pirámide y en los resultados recolectados de los artículos revisados.

\section{Tabla I}

\section{Beneficios de la estimulación vestibular en el desarrollo infantil}

\begin{tabular}{|c|c|c|c|}
\hline Población & & Edad & Beneficios de estimulación vestibular \\
\hline Prematuro & Sano & 1er año & $\begin{array}{l}\text { - Relajación (contención) } \\
\text { - Alerta visual } \\
\text { - Activación cuando se trata de aumentar tono } \\
\text { - Ganancia ponderal } \\
\text { - Mejoría en el tono muscular pasivo } \\
\text { - Maduración de patrones de sueño } \\
\text { - Reducción de periodos de apnea } \\
\text { - Regulación de la respiración }\end{array}$ \\
\hline \multirow{3}{*}{$\begin{array}{c}\text { Trastornos de desarrollo } \\
\text { neurológicos } \\
\text { (Trastornos de desarrollo } \\
\text { intelectual, } \\
\text { trastornos de comunicación, } \\
\text { trastorno del espectro autista, } \\
\text { trastorno por déficit de aten- } \\
\text { ción con hiperactividad, } \\
\text { trastorno específico del } \\
\text { aprendizaje, } \\
\text { trastornos motores) }\end{array}$} & \multirow{3}{*}{ Sano } & 1-3 años & $\begin{array}{l}\text { - Ejecución de praxis } \\
\text { - Esquema corporal } \\
\text { - Lateralidad } \\
\text { - Equilibrio y coordinación dinámica general }\end{array}$ \\
\hline & & 3-6 años & $\begin{array}{l}\text { - Lecto- escritura } \\
\text { - Conceptos numéricos } \\
\text { - Habilidades visoperceptuales } \\
\text { - Habilidades matemáticas }\end{array}$ \\
\hline & & 6-12 años & - Conducta adaptativa \\
\hline
\end{tabular}




\section{Conclusiones}

La estimulación del sistema vestibular constituye un recurso esencial e indispensable para la óptima interacción con el medio. Resulta muy accesible ya que no es necesario el uso de materiales costosos y es sencillo de aplicar. A través de ésta se optimiza y facilita la comunicación con el cuidador primario, quien provee nuevas experiencias al niño, siendo posible entonces coadyuvar en el desarrollo psicomotor a través de la interacción dependiente de experiencias enriquecedoras entre el facilitador adulto y el niño, lo que condiciona un escenario óptimo para el desarrollo positivo en la infancia.

El ambiente dentro del hogar explica parcialmente el porvenir del desarrollo, dado que aquellos niños, en un ambiente enriquecido, presentan un mejor desarroIlo, y mayores posibilidades de disminución de la expresión de alguna discapacidad cuando el ambiente está mediado por una supervisión constante y las personas involucradas en su desarrollo llevan a cabo estrategias diseñadas específicamente para estimular el paso al siguiente nivel a desarrollar. De lo contrario, el niño muy probablemente se verá afectado en su neurodesarrollo; esto, sin un buen diagnóstico o seguimiento, repercute en la salud y desarrollo del niño, por ejemplo, desde una perspectiva social, puede verse afectado en su integración y ubicación dentro del aula escolar. De igual manera puede llegar a alterar la dinámica y economía familiar por el tiempo, los costos de la atención y cuidados especiales que pueden requerirse.

Es importante destacar la conveniencia de contar con personal de salud entrenados para realizar tamizajes eficientes en población de riesgo y docentes con bases pedagógicas que identifiquen tempranamente alteraciones en el procesamiento sensorial, a través de signos clínicos o estudios de imagen, para la constitución de planes de intervención a través de estrategias dirigidas a estimular de manera efectiva a grupos vulnerables y marginados, que tengan mayor impacto y faciliten la funcionalidad de éstos, se podrá observar una importante disminución en los índices de discapacidad. Por esto la importancia de la elaboración de nuevas estrategias de estimulación vestibular para niños de diferentes grupos de edad, promoviendo el desarrollo infantil.

\section{AGRADECIMIENTOS}

Los autores de este artículo agradecen el apoyo a las siguientes personas por el apoyo para su elaboración: Dra. Thalía Harmony Baillet, Dra. María Elena Juárez Colin, José Francisco Vázquez Mellado Larracoechea, MTI. Ana Cecilia Vázquez Mellado Larracoechea, LTF Asahel Martínez Carreño, PSS. Daniel Pérez Arreguin, PSS. Andrea Lucas Galindo, PSS. Daniela Trejo Méndez, PSS. Sharon Pedroza Vargas, LTF. Ana Karen Santos Chávez, Lic. Psic. Mireya Capistrán Martínez. 


\section{Bibliografía}

1. Delval J. El desarrollo humano. Madrid: Siglo Veintiuno Editores; 2002

2. Piaget J. Seis Estudios De Psicología. Cuadernos Políticos. Barcelona: Editorial Labor; 1991. 199 p.

3. Fonseca $\vee$ da. Manual de observación psicomotriz: Significación psiconeurológica de los factores psicomotores. Barcelona: INDE publicaciones; 1998. 382 p.

4. Bennet L, Van Den Heuij L, M Dean J, Drury P, Wassink G, Jan Gunn A. Neural plasticity and the Kennard principle: Does it work for the preterm brain? Clin Exp Pharmacol Physiol. 2013;40(11):774-84.

5. Dennis M. Margaret Kennard (1899-1975): Not a "Principle" of brain plasticity but a founding mother of developmental neuropsychology. Cortex. 2010;46(8):1043-59.

6. Doman G. What to do About Your Brain-Injured Child. 12o. Filadelfia: Diana; 2009.

7. Raine S, Meadows L, Lynch-Ellerington M. Bobath Concepts: Theory and clinical practice in neurological rehabilitation. Chichester: Wiley-Blackwell; 2009.

8. Ayres J. La integración sensorial y el niño. 2nd ed. México: Trillas; 2016. 226 p.

9. Alvarado-Ruíz G, Martínez- Vázquez I, Sánchez C, Solís-Chan M, Mandujano M. Los movimientos elementales complejos del humano. Desarrollo postnatal. Reporte preliminar de nueve lactantes mexicanos. Salud Ment. 2012;35(2):99-107.

10. Pelayo HJ, Solovieva Y, Quintanar L, Reyes V. Efectos de la estimulación del neurodesarrollo en niños con antecedentes de encefalopatía hipóxico isquémica. Pensam Psicológico [Internet]. 2014;12(1):1121. Disponible en: http://revistas.javerianacali. edu.co/index.php/pensamientopsicologico/article/ view/690

11. Porras-Kattz E, Harmony T. Neurohabilitación: un método diagnóstico y terapéutico para prevenir secuelas por lesión cerebral en el recién nacido y el lactante. Bol Med Hosp Infant Mex [Internet]. 2007;64(2):125-35. Disponible en: http://www.scielo.org. $\mathrm{mx} /$ scielo.php?script $=\mathrm{sci}$ arttext\&pid $=$ S1665-11462007000200008\&lng $=\overline{p t}$ $\& n r m=i s o \& t \operatorname{lng}=e s$

12. Roberts RE, Ahmad $H$, Arshad $Q$, Patel M, Dima $D$, Leech $R$, et al. Functional neuroimaging of visuovestibular interaction. Brain Struct Funct [Internet]. 2017 Jul 10;222(5):2329-43. Disponible en https://www.ncbi.nlm.nih.gov/pubmed/27942855

13. Hinojosa-Rodríguez $M$, Harmony $T$, Carrillo-Prado C, Van Horn JD, Irimia A, Torgerson C, et al. Clinical neuroimaging in the preterm infant: Diagnosis and prognosis. Neurolmage Clin [Internet]. 2017;16:355-68. Disponible en: https://linkinghub. elsevier.com/retrieve/pii/S2213158217302061

14. Ottenbacher K. Developmental implications of clinically applied vestibular stimulation. Phys Ther. 1983;63(3):338-42.

15. Cano R, Molero A, Carratalá M. Teorías y modelos de control y aprendizaje motor. Aplicaciones clínicas en neurorehabilitación. Neurología. 2015;30(1):3241.
16. Quiroz P. ¿Cómo lograr un desarrollo infantil de calidad? Factores de éxito para una atención integral. In: I Congreso Internacional de Neuroeducación y Primera Infancia y Mesa de Responsabilidad Social Empresaria. Córdoba: Organización de Estados Americanos; 2012.

17. Macías L, Fagoaga J. Fisioterapia en pediatría. 1a. McGraw Hill-Interamericana; 2002. 461 p.

18. Arendt RE, Maclean WE, Halpern LF, Youngquist $\mathrm{GA}$, Baumeister AA. The influence of rotary vestibular stimulation upon motor development of nonhandicapped and down syndrome infants. Res Dev Disabil [Internet]. 1991 Jan;12(3):333-48. Disponible en: http://linkinghub.elsevier.com/retrieve/ pii/089142229190016L

19. Volpe J. Neurology of the Newborn. 5th ed. Philadelphia: Saunders Elsevier; 2008.

20. Cano R, Martínez RM, Miangolarra JC. Control y Aprendizaje motor: Fundamentos, desarrollo y reeducación del movimiento humano. Madrid: Editorial Médica Panamericana; 2017.

21. Molina T, Banguero LF. Diseño de un espacio sensorial para la estimulación temprana de niños con multidéficit. Rev Ing Biomédica [Internet]. 2008;2(3):407. Disponible en: http://www.scielo.org.co/pdf/ rinbi/v2n3/v2n3a07

22. Medina $M$ del $P$, Caro I, Muñoz $P$, Leyva J, Moreno J, Vega SM. Neurodesarrollo Infantil: Características normales y signos de alarma en el niño menor de cinco años. Rev Peru Med Exp Salud Publica [Internet] 2015;32(3):565-73. Disponible en: http://www.redalyc.org/articulo.oa?id=36342789022

23. Lázaro A, Blasco S, Lagranja A. La integración sensorial en el Aula Multisensorial y de Relajación: estudio de dos casos. Rev Electrónica Interuniv Form del Profr [Internet]. 2010;13(4):321-34. Disponible en: http://www.redalyc.org/articulo. oa? id $=217015570027$

24. Lázaro A, Berruezo PP. La pirámide del desarrollo humano. Rev Iberoam Psicomot y Técnicas Corporales [Internet]. 2009;9(34):15-42. Disponible en: https://dialnet.unirioja.es/servlet/ articulo? codigo $=3660083$

25. American Psychiatric Association. Guía de consulta de los criterios diagnósticos del DSM- $5 \circledR$ [Internet]. American Psychiatric Publishing; 2014. 492 p. Disponible en: http://psychiatryonline.org/doi/ book/10.1176/appi.books.9780890425657

26. Braswell J. Recognizing Vestibular Problems in Children [Internet]. Neurology Section; 2010. Disponible en: http://www.neuropt.org/docs/vsig-english-ptfact-sheets/recognizing-vestibular-problems-in-children.pdf?sfvrsn=ac776558_2

27. Torricelli R. Vértigo en el niño. Anatomía y fisiología del sistema vestibular Evaluación del paciente. Med (Buenos Aires) [Internet]. 2007;67(6):631-8. Disponible en: http://www.scielo.org.ar/scielo.php?script=sci arttext\&pid=S0025-76802007000700012

28. Wiener-Vacher S, Hamilton D, Wiener S. Vestibular activity and cognitive development in children: perspectives. Front Integr Neurosci [Internet]. 2013;7:113. Disponible en: https://www.ncbi.nlm.nih.gov/ pubmed/24376403

Número cuarenta, enero-abril 2019 LUX MÉDICA 
29. Erazo O. Identificación, Descripción y relaciones entre la Integración Sensorial, Atención y Conducta. Rev Colomb Ciencias Soc [Internet]. 2016;7(1):2148. Disponible en: https://dialnet.unirioja.es/servlet/articulo?codigo $=5454154$

30. Serna S, Torres K, Torres MA. Desórdenes en el procesamiento sensorial y el aprendizaje de niños preescolares y escolares: Revisión de la literatura. Rev Chil Ter Ocup. 2017;17(2):81-90.

31. Lázaro A. Estimulación vestibular en Educación Infantil. Rev Interuniv Form del Profr [Internet]. 2008;62(22):165-74. Disponible en: http://www. redalyc.org/articulo.oa?id $=27414780010$

32. Secretaría de Salud. En México nacen cada año 200 mil niños prematuros: INP. In: $4^{\circ}$ Simposio Nacional del Día del Prematuro [Internet]. México; 2015. Disponible en: https://www.gob.mx/salud/prensa/enmexico-nacen-cada-ano-200-mil-ninos-prematurosinp

33. Zimmerman E, Barlow S. The Effects of Vestibular Stimulation Rate and Magnitude of Acceleration on Central Pattern Generation For Chest Wall Kinematics in Preterm Infants. J Perinatol. 2013;32(8):61420.

34. Clark D, Cordero L, Goss K, Manos D. Effects of Rocking on Neuromuscular Development in the Premature. Biol Neonate. 1989;56(6):306-14.

35. Barreras J, Guerra A. Programa de intervención temprana para la prevención de la minusvalía neurológica en niños de alto riesgo de la provincia
Camagüey. Rev Arch Médico Camagüey [Internet]. 2002;6(3):217-21. Disponible en: http:// scielo.sld.cu/scielo.php?script $=$ sci_arttext $\&$ pid $=$ S1025-02552002000300001

36. Pérez- Olarte P. Evaluación y manejo del niño con retraso psicomotor. Pediatría Integr. 2003;VII(8):55766.

37. Sallustro F, Atwell CW. Body rocking, head banging, and head rolling in normal children. J Pediatr. 1978;93(4):704-8.

38. Cordero L, Clark D, Schott L. Effects of Vestibular Stimulation on Sleep States in Premature Infants. Am J Perinatol [Internet]. 1986 Oct 4;3(04):319-24. Disponible en: http://www.thieme-connect.de/DOI/ DOI?10.1055/s-2007-999888

39. Neal M. Vestibular Stimulation and Development of the Small Premature Infant. Commun Nurs Res [Internet]. 1977;8:291-302. Disponible en: https:// www.ncbi.nlm.nih.gov/pubmed/585604

40. Gregg CL, Haffner ME, Korner AF. The Relative Efficacy of Vestibular-Proprioceptive Stimulation and the Upright Position in Enhancing Visual Pursuit in Neonates. Child Dev [Internet]. 1976;47(2):309-14. Disponible en: https://www.ncbi.nlm.nih.gov/pubmed/1083792

41. Korner AF, Thoman EB. The Relative Efficacy of Contact and Vestibular-Proprioceptive Stimulation in Soothing Neonates. Child Dev [Internet]. 1972 Jun;43(2):443. Disponible en: https://www.ncbi. nlm.nih.gov/pubmed/4537551 